Tropical Journal of Pharmaceutical Research September 2017; 16 (9): 2069-2077

ISSN: 1596-5996 (print); 1596-9827 (electronic)

(C) Pharmacotherapy Group, Faculty of Pharmacy, University of Benin, Benin City, 300001 Nigeria.

All rights reserved.

Available online at http://www.tjpr.org

Original Research Article

http://dx.doi.org/10.4314/tjpr.v16i9.5

\title{
Stability and drug dissolution evaluation of Qingkailing soft/hard capsules based on multi-component quantification and fingerprint pattern statistical analysis
}

\author{
Ruyu Sun, Huihui Teng, Xiaonan Chen, Shuang Guo, Shan Jia, Mengcheng \\ Zheng, Yang Lu, Jie Bai, Pengyue Li* and Shouying Du \\ School of Chinese Materia Medica, Beijing University of Chinese Medicine, Beijing 100102, China
}

*For correspondence: Email: dushouying@263.net; Tel/Fax: +86 01084738615

Sent for review: 19 April 2017

Revised accepted: 28 August 2017

\begin{abstract}
Purpose: To carry out a post-marketing evaluation of the stability and drug dissolution of Qingkailing soft/hard capsules.

Methods: High performance liquid chromatography with diode array detection (HPLC-DAD) method was developed for the determination of three key ingredients (chlorogenic acid, geniposide and baicalin) and fingerprints of QKL soft/hard capsules. Stability tests were carried out based on long-term testing. The drug release profile of Qingkailing soft and hard capsules were studied using semi-bionic incubation experiments.

Results: The linearity, precision, stability, repeatability and recovery of HPLC and fingerprint all met the requirements of CFDA. Stability data from long-term studies showed that within 6 months the contents of the three key ingredients in both soft and hard capsules remained $>90 \%$. However, fingerprint pattern statistical analysis showed that the soft capsule is more stable than the hard capsule. Furthermore, the key ingredients of the hard capsule dissolved much faster $(p<0.05)$ than from the soft capsule. The level of dissolved drug of hard capsule is about 4 times the rate of soft capsule, after a 4- $h$ incubation in gastric lavage fluid. In intestinal lavage fluid, more than $90 \%$ of chlorogenic acid, geniposide and baicalin of hard capsule were dissolved in $2 h$, while the soft capsule displayed a $12 h$ sustained release. Fingerprint pattern statistical analysis also showed that most of the components of soft capsule dissolved after $8 \mathrm{~h}$.

Conclusion: Compared with the hard capsule, Qingkailing soft capsule has certain advantages in stability and drug dissolution, which may affect the biopharmaceutics and the clinical effects of the drug.
\end{abstract}

Keywords: Qingkailing capsule, Chlorogenic acid, Geniposide, Baicalin, Fingerprint, Sustained release, Principal component analysis

Tropical Journal of Pharmaceutical Research is indexed by Science Citation Index (SciSearch), Scopus, International Pharmaceutical Abstract, Chemical Abstracts, Embase, Index Copernicus, EBSCO, African Index Medicus, JournalSeek, Journal Citation Reports/Science Edition, Directory of Open Access Journals (DOAJ), African Journal Online, Bioline International, Open-J-Gate and Pharmacy Abstracts

\section{INTRODUCTION}

Qingkailing (QKL) is a famous herbal compound prescription in China, which consists of Radix isatidis, Flos lonicerae, Fructus gardeniae, Bubali cornu, Concha margaritifera, baicalin, cholic acid and hyodeoxycholic acid. QKL injection is widely used clinically for the treatment of cerebrovascular ischemic disease, ardent fever, hepatitis, pneumonia, $\mathrm{H} 1 \mathrm{~N} 1$, and avian influenza. However, with the increase of ADR cases reported these years [1], capsule is favored by more and more patients and doctors in China. There are two marketed forms of QKL capsules in China, hard and soft capsule, with annual 
sales exceeding RMB 1 billion (US\$ 0.15 billion) in 2016.

A previous study has shown that the bioavailability of baicalin in soft capsule is higher than that of hard capsule [2]. Compared with hard capsule, soft capsule had a longer mean residence time (MRT), a higher maximum concentration $\left(\mathrm{C}_{\max }\right)$, and a larger area under the curve (AUC). The improvement may be caused by the dosage form. Soft capsule may be more beneficial to the stability of the active ingredients both in vitro and in vivo. The drug dissolution behavior may also affect the pharmacokinetic behavior in vivo.

Therefore, the purpose of this study was to evaluate the stability and the drug dissolution behavior of the two capsules by long-term stability testing and semi-bionic incubation experiments.

\section{EXPERIMENTAL}

\section{Materials and processing software}

Standards of chlorogenic acid, baicalin and geniposide were obtained from National Institutes for Food and Drug Control (Beijing, China); methanol, acetonitrile and phosphoric acid of HPLC grade were obtained from Thermo Fisher Scientific Inc. (USA); pure water of HPLC grade was purchased from Wahaha (Hangzhou Wahaha Group Co.,Ltd.); QKL soft capsules were offered by Shineway Pharmaceutical Group Co. Ltd in China (batch number: 14072411), QKL hard capsule were offered by Guangzhou Baiyunshan Mingxing Pharmaceatical Co. Ltd in China (batch no. 140512).

\section{Animals}

Male Sprague-Dawley rats $(220-250 \mathrm{~g})$ of SPF grade were purchased from Vital River Laboratory Animal Technology Co. Ltd, China. Before experiment, the animals were fasted 24 hours, with free access to water. The experimental protocols were conducted in accordance with The Guiding Principles for the Care and Use of Laboratory Animal [3] in China. Experimental protocols were approved by the institutional animal experimentation committee of Beijing University of Chinese Medicine (approval ref. no. : Kj-dw-18- 20150923-01).

The fingerprint data were processed by principal component analysis (PCA) using SIMCA-P 11.5 software (Umetrics $A B$, Sweden).
Evaluation of long-term stability of soft and hard capsules

The soft/hard capsules were placed for $0,1,2,3$, 6 month as stated in Chinese Pharmacopoeia. The test was carried out at the beginning of each month. Three key ingredients (chlorogenic acid, geniposide and baicalin) were determined according to the method established.

\section{Pretreatment of QKL soft capsules}

Oily content $(0.4 \mathrm{~g})$ of QKL soft capsules was absorbed by diatomite $(1.2 \mathrm{~g})$ and the mixture was transferred into a flask $(25 \mathrm{ml})$ and solvent (acetonitrile: $0.08 \%$ phosphoric acid, 39:61) was added in to volume. The flask was then weighted accurately followed by ultrasonic extraction (200 w, $49 \mathrm{kHz}, 70 \mathrm{~min}$ ). After that, the same solvent was added in to make up the solvent loss caused by ultrasonic extraction. A portion of this solution was centrifugated for $10 \mathrm{~min}(10,000 \mathrm{rpm})$. Then pass the supernatant through a filter having a $0.45-\mu \mathrm{m}$ porosity, and use the filtrate after discarding the first $2 \mathrm{ml}$. Two samples were prepared by the same method at a time.

\section{Pretreatment of QKL hard capsules}

Transfer an accurately weighed portion of the powder $(0.5 \mathrm{~g})$ of hard capsules to a 25 -ml flask, solvent (acetonitrile: $0.08 \%$ phosphoric acid, 39:61) was added in to volume. The flask was weighted accurately followed by ultrasonic extraction, the remaining steps were the same as soft capsule group. Two samples were prepared by the same method at a time.

\section{Fingerprint analysis}

The fingerprint of the two capsules was studied according to the Technical Requirements of Fingerprint Research on Traditional Chinese Medicine Injection [4], which is made by CFDA. Baicalin, the highest peak, was chosen as the reference peak. The precision, stability and repeatability were studied and were all met the requirements of the guidance.

\section{Drug dissolution studies by semi-bionic incubation methods}

Simulated gastric fluid (SGF) and simulated intestinal fluid (SIF) were prepared according to Chinese Pharmacopoeia [5]. Preparation of gastric lavage fluid: Rats fasted $24 \mathrm{~h}$ with free access to water. They were anaesthetized by 20 $\%$ urethanes $(2 \mathrm{ml})$ and placed on a hot pad to maintain normal body temperature. An abdominal midline incision was made. Then 
semi-circular incisions were made at the ends of stomach. PE tubes were inserted into cardia and pylori respectively and then ligated with silk sutures. Stomach was lavaged with $15 \mathrm{ml}$ SGF $\left(37^{\circ} \mathrm{C}\right)$. The lavage fluid of 3 rats (about $40 \mathrm{ml}$ ) was collected into a $50 \mathrm{ml}$ flask and then dilute with SGF to volume, and mix.

Preparation of intestinal lavage fluid: Rats were anaesthetized and were celiotomized. Semicircular incisions were made at the fore-end of duodenum and back-end of ileum. PE tubes were inserted in and ligated with silk sutures. Small intestine was lavaged with $40 \mathrm{ml}$ SIF (37 $\left.{ }^{\circ} \mathrm{C}\right)$. The lavage fluid was collected into a $50 \mathrm{ml}$ flask and then dilute with SIF to volume, and mix.

Drug dissolution in gastric lavage fluid: Place 50 $\mathrm{ml}$ gastric lavage fluid in a triangular flask, equilibrate the dissolution medium to $37^{\circ} \mathrm{C}$ in a constant temperature shaker. Place QKL soft capsule (or hard capsule) in the triangular flask. At each of the test-time points $(1,5,10,30,60$, 90,120 and $240 \mathrm{~min}$ ), withdraw a specimen (1 $\mathrm{ml}$ ) from the triangular flask. And replace the aliquots withdrawn for analysis with equal volumes of fresh dissolution medium at $37^{\circ} \mathrm{C}$. Sodium hydroxide solution $(0.1 \mathrm{~mol} / \mathrm{l}, 400 \mu \mathrm{l})$ was added into the withdrawn as the neutralizer. Then, add $2 \mathrm{ml}$ of methanol to precipitate the pepsin.

The mixture was vortexed for $3 \mathrm{~min}$ and then centrifuged for $10 \mathrm{~min}(10,000 \mathrm{rpm})$. Pass the supernatant through a filter having a $0.22-\mu \mathrm{m}$ porosity, and use the filtrate after discarding the first $0.5 \mathrm{ml}$ as the test solution. Inject $20 \mu \mathrm{l}$ test solution into the chromatograph, record the chromatograms, and measure the peak responses. Six dosage units were tested by the same method at a time. The amount of dissolved drug $Q$ (expressed as a percentage of the labeled content of the dosage unit) was calculated according to $\mathrm{Eq} 1$.

$\mathrm{Q}_{\mathrm{n}}=\frac{3.4 \times 50 \times C_{n}+\sum_{i=1}^{n-1} C_{i} \times 3.4 \times 1}{D}$

where $\mathrm{C}_{\mathrm{n}}$ is the drug concentration of the nth time point $(\mathrm{mg} / \mathrm{ml}) ; \mathrm{C}_{i}$ is the drug concentration in flask of the ith ( $i \leq n-1$ ) sampling point; $D$ is the content of the dosage unit.

Drug dissolution in intestinal lavage fluid: Both hard capsule and soft capsule broke in the gastric lavage fluid in a few minutes. In this study, the contents of capsules (instead of capsules) were incubated with intestinal lavage fluid $(50 \mathrm{ml})$. The test-time points were 0.083,
0.25, 0.5, 1, 1.5, 2, 4, 6, 8, 12, 18, 24 h. At each of the time stated, withdraw a specimen $(1 \mathrm{ml})$ from the triangular flask. Then, add $2 \mathrm{ml}$ of methanol to precipitate trypsin, the remaining steps were the same as gastric lavage fluid group. The amount of dissolved drug $Q$ was calculated according to Eq 2.

$\mathrm{Q}_{\mathrm{n}}=\frac{3 \times 50 \times C_{n}+\sum_{i=1}^{n-1} C_{i} \times 3 \times 1}{D}$

where $\mathrm{C}_{\mathrm{n}}$ is drug concentration of the nth time point $(\mathrm{mg} / \mathrm{ml}) ; C_{i}$ is the drug concentration in flask of the ith ( $i \leq n-1$ ) sampling point; $D$ is the content of the dosage unit.

The linearity, precision, stability, repeatability and recovery of chlorogenic acid, geniposide, baicalin were studied according to General Requirements of Chinese Pharmacopoeia [5]. The fingerprint methodology was studied according to Technical Requirements of Fingerprint Research on Traditional Chinese Medicine Injection [4]. And they all met the requirements.

\section{Chromatographic conditions}

The separation conditions were carried out on a Phenomenex Luna RP18 column $(250 \times 4.6 \mathrm{~mm}$ i.d., $5 \mu \mathrm{m})$, the temperature was set at $30{ }^{\circ} \mathrm{C}$. 0.4 $\%$ phosphoric acid in water (phase A) and methanol-Phosphoric acid (4:1) (phase B) were used as mobile phase under the gradient elution mode: A:B (v/v) 95:5 (0 min); 70:30 (15 min); 65:35 (22 min); 40:60 (40 min), 40:60 (43 min); 19:81 (55 min); 10:90 (60 min), and 10:90 (65 $\min )$.

The flow rate was set at $1.0 \mathrm{ml} / \mathrm{min}$. A DAD detector was used for detection, and the wavelength was set at $327 \mathrm{~nm}$ (for chlorogenic acid), $240 \mathrm{~nm}$ (for geniposide), $278 \mathrm{~nm}$ (for baicalin), $254 \mathrm{~nm}$ (for fingerprint).

\section{Data analysis}

The contents of three key ingredients were calculated according to the peak responses of standards and samples. Data of soft capsule and hard capsule groups were analyzed by Student's test with $p<0.05$ set as statistically different.

Fingerprint data of different capsules or different sampling time points were imported to SIMCA-P (version 11.5) software and were processed by principal component analysis (PCA). It is a wellknown chemometric method for the decomposition of two-dimensional matrices. Loading $\mathrm{Bi}$ 
Plots can be offered by the software without manual calculation.

\section{RESULTS}

Long-term stability comparison between soft and hard capsules based on multi-component quantification

The contents of chlorogenic acid, geniposide and baicalin in hard capsule and soft capsule were determined. The contents of three key ingredients remained were shown in Figure 1. As was shown in the picture, these key ingredients degraded slowly in the past 6 months in both soft and hard capsules, but they all remained $>90 \%$. No differences were observed $(p>0.05)$.
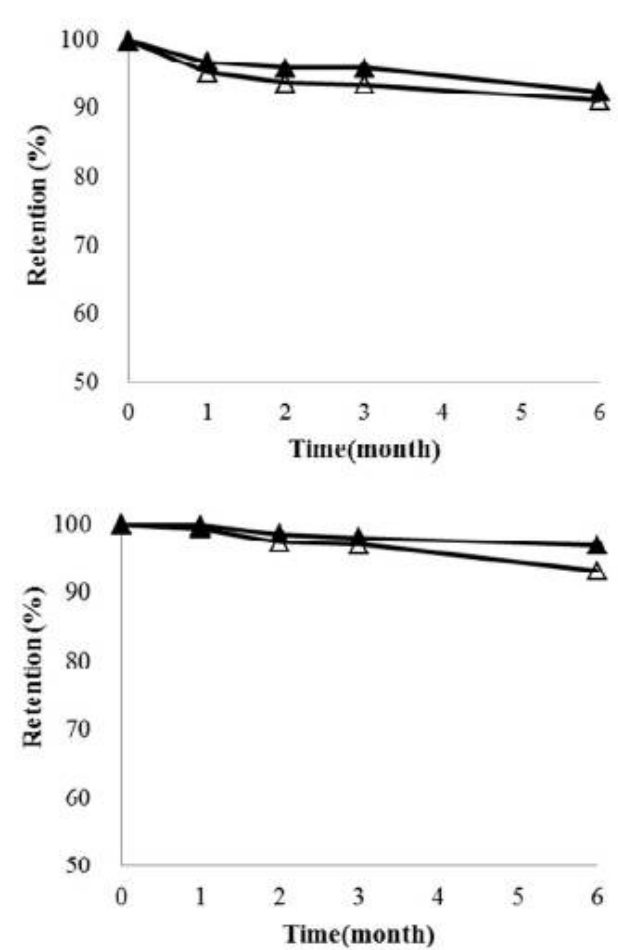

B

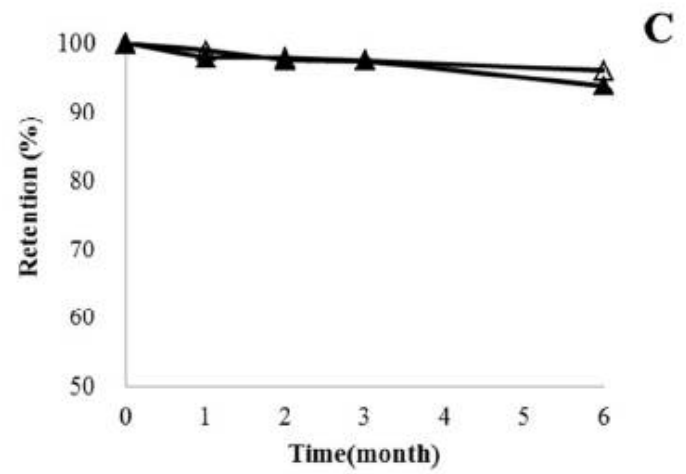

Figure 1: Long-term stability test of 3 key ingredients in QKL soft/hard capsules. $A=$ chlorogenic acid; $B=$ geniposide; $C$ = baicalin; $\Delta$ : soft capsule; $\mathbf{\Delta}$ : hard capsule
Long-term stability of soft and hard capsules based on fingerprint pattern analysis

There are many other ingredients in this herbal compound prescription. There were 21 common peaks (their peak areas account for $87.42 \%$ of all peaks areas) and 16 common peaks (their peak areas account for $84.59 \%$ of all peaks areas) were identified in soft and hard capsules samples (11 batches for each capsule), respectively.

PCA was used to evaluate the stability of two capsules integrally. Fingerprint data of QKL soft/hard capsules were imported in software SIMCA-P 11.5. And PC1 could explain nearly $100 \%$ of variance.

t[Comp. 1]/t[Comp. 2]

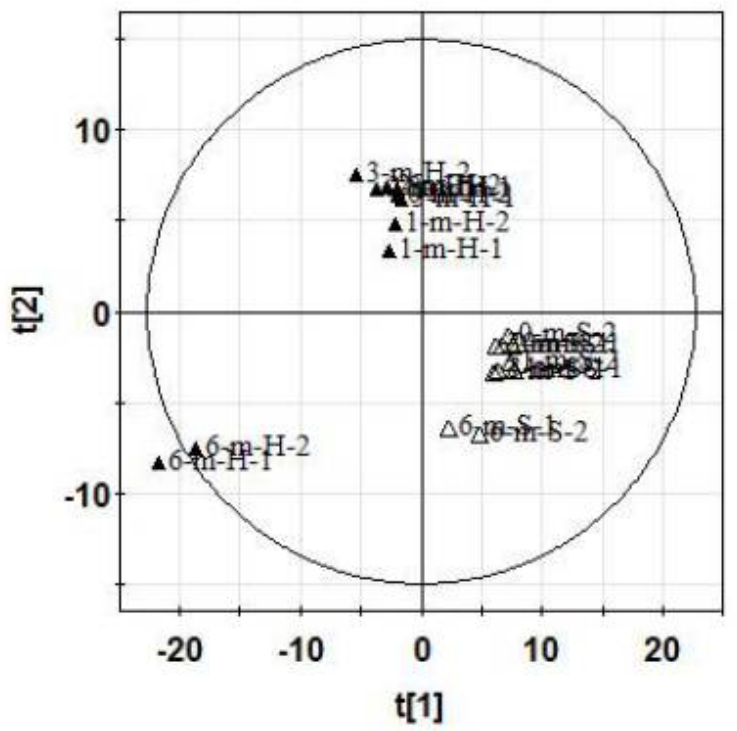

Figure 2: PCA scatter plot of $Q K L$ soft and hard capsules. $\mathbf{\Delta}$ : hard capsules of different month ; $\Delta$ : soft capsules of different month

The PCA scatter plot (PC1 versus PC2) was shown in Fig 2. The samples of soft capsules of $0,1,2,3,6$ month (two samples for each time point) were clustered together. But, the samples of hard capsules were clearly divided into two groups, which indicated that samples of 6 month were quite different from the other samples of hard capsule.

Drug dissolution of QKL soft and hard capsules in gastric lavage fluid

QKL soft and hard capsules ruptured in no more than 15 minutes and 1 minute, respectively. As was shown in Figure 3, for hard capsule, not less than $50 \%(Q)$ of the chlorogenic acid and geniposide were dissolved in $15 \mathrm{~min}$, and they kept growing in the next $225 \mathrm{~min}$. But the $\mathrm{Q}$ of 
baicalin decreased after a fast dissolution in the first $15 \mathrm{~min}$.

For soft capsule group, the $Q$ of three ingredients kept growing in $240 \mathrm{~min}$, and finally reached 7.32 $\%$ (chlorogenic acid), $13.64 \%$ (geniposide), 1.02 $\%$ (baicalin).
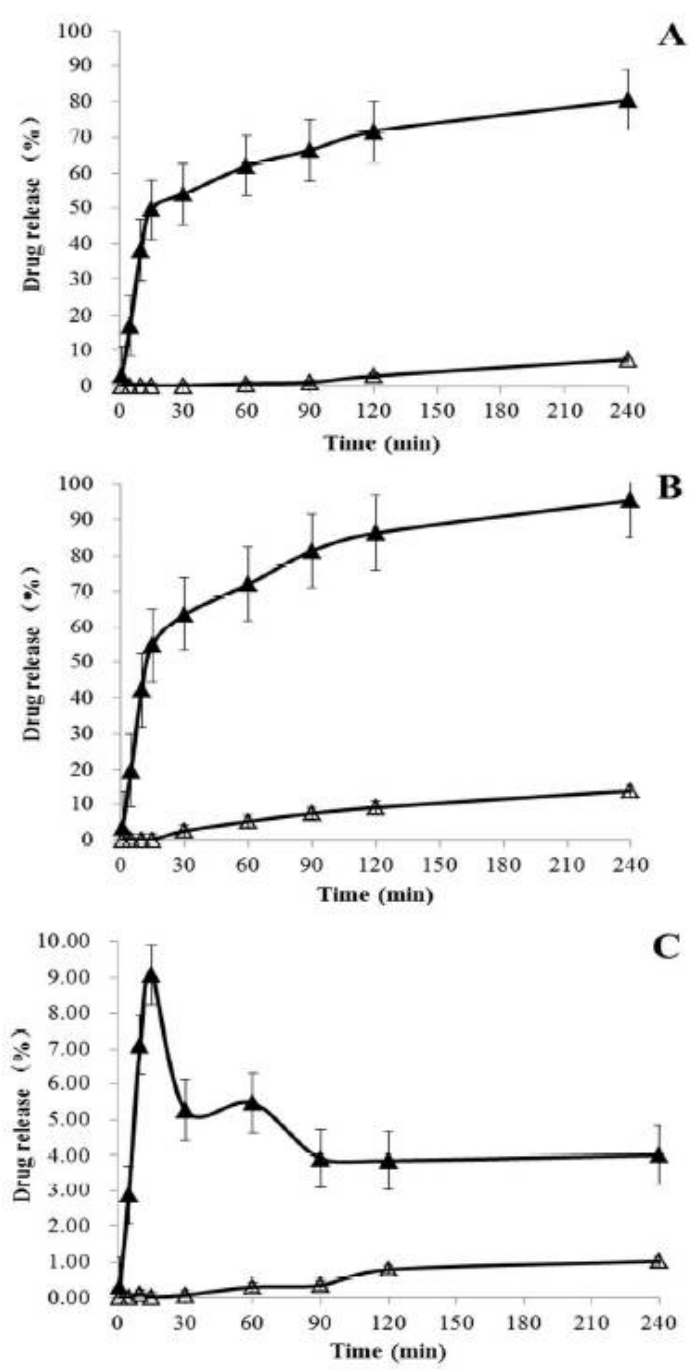

Figure 3: Drug dissolution of 3 key ingredients of $\mathrm{QKL}$ soft/hard capsules in gastric lavage fluid. $A=$ chlorogenic acid; $B=$ geniposide; $C=$ baicalin; $\Delta$ : soft capsule; $\boldsymbol{\Delta}$ : hard capsule

\section{Drug dissolution of QKL soft and hard capsules in intestinal lavage fluid}

Compared with gastric lavage fluid group, different dissolution behavior was observed in intestinal lavage fluid. As was shown in Figure 4, for hard capsule, not less than $90 \%$ of chlorogenic acid, geniposide and baicalin were dissolved in $2 \mathrm{~h}$. Then, they all declined gently (to about $80 \%$ ).

However, for soft capsule, whose dissolution behavior is quite different, the $Q$ of 3 ingredients kept growing up to $95.18 \%$ (chlorogenic acid), $93.18 \%$ (geniposide), $99.31 \%$ (baicalin) in $12 \mathrm{~h}$. And they were stable in the following $12 \mathrm{~h}$ (with a variation of less than $3 \%$ ).
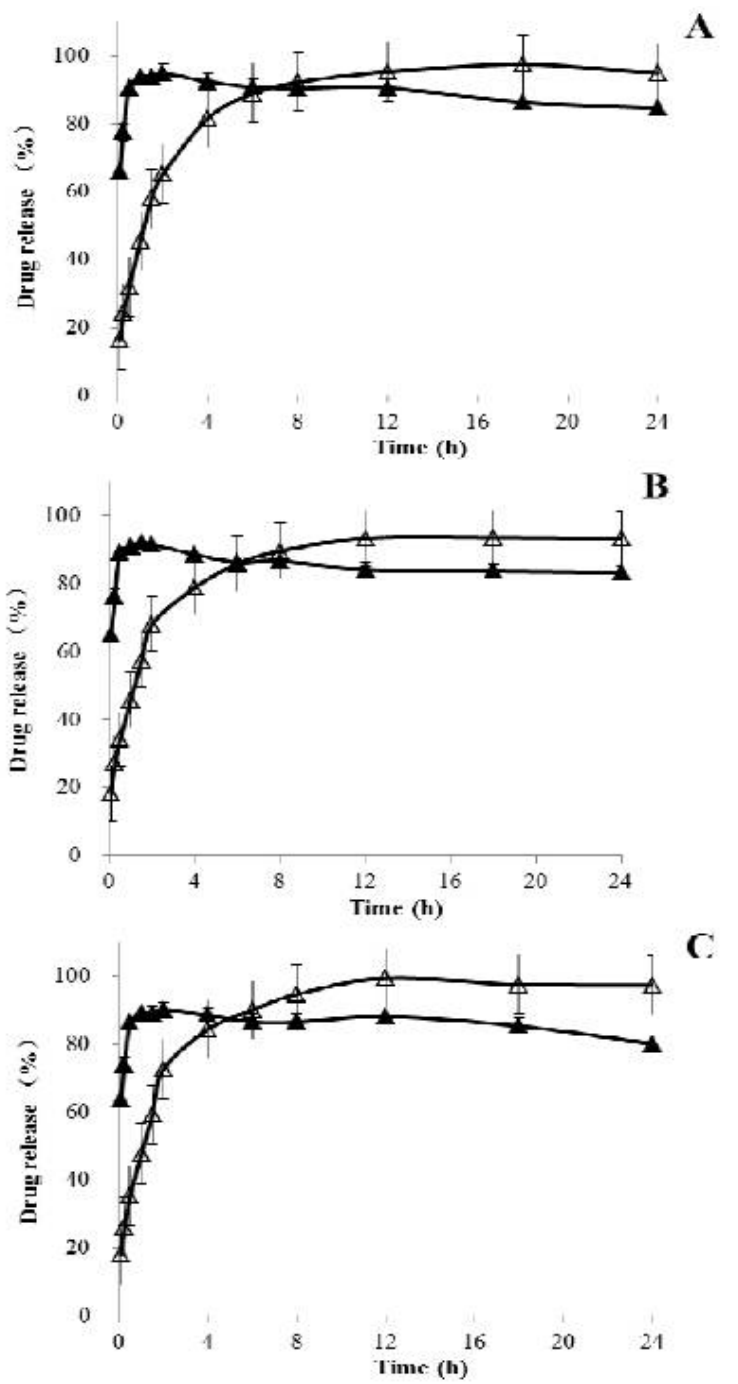

Figure 4: Drug dissolution of 3 key ingredients of QKL soft/hard capsules in intestinal lavage fluid. $A=$ chlorogenic acid; $B=$ geniposide; $C=$ baicalin; $\Delta$ : soft capsule; $\boldsymbol{\Delta}$ : hard capsule

Drug dissolution of QKL soft capsules in intestinal lavage fluid based on fingerprint pattern analysis

As shown in Figure 3 and Figure 4, the key ingredients were unstable when hard capsules were incubated with gastric lavage fluid and intestinal lavage fluid. The degradation may affect the analysis based on fingerprint pattern statistical analysis. However, soft capsule can keep the ingredients stable in intestinal lavage fluid, the $Q$ of 3 ingredients were kept at about 95 $\%$ finally. So, drug dissolution behavior of soft capsule in intestinal lavage fluid based on fingerprint pattern analysis was studied. 
PCA was also used to evaluate the drug dissolution behavior of soft capsules integrally. Fingerprint data of QKL soft capsules were imported in software (six samples for each time point).

For soft capsules, three principal components affecting the dissolution behavior were identified with a cumulative score $43.7 \%$. The loading $\mathrm{Bi}$ Plot (PC1 versus PC2) was shown in Figure 5. Chromatogram peaks $(\Delta)$ close to the time points (घ) contribute the most to the differentiation of the samples of different sampling time, which indicated that, for soft capsule, peak 11, 18, 19, 23, 24, 26, 31, 32, 41, 46, 74 dissolved before 1 h, peak $8,25,28,30,40,43,59$ dissolved from 1.5 to 2 h, peak $2,4,6,7,10,15,17,20,29,33$, $34,37,38,42,44,47,49,51,56,58,62,70,71$, 73 dissolved from 2 to $4 \mathrm{~h}$, the other peaks dissolved after $6 \mathrm{~h}$.

p(corr) [Comp. 1]/p(corr)[Comp. 2]

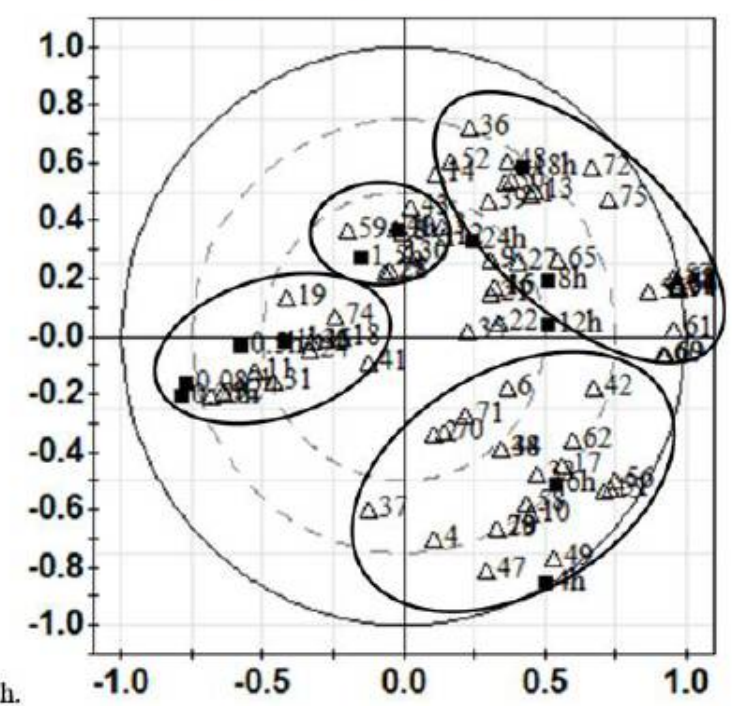

Figure 5: Loading Bi Plot of QKL soft capsules' dissolution behavior in intestinal lavage fluid. a: different time points for sampling $(0.083,0.25,0.5,1$, 1.5, 2, 4, 6, 8, 12, 18, $24 \mathrm{~h}) ; \Delta$ : Chromatogram peaks of QKL soft capsules

\section{DISCUSSION}

The key ingredients of QKL soft/hard capsules had good stability in vitro within 6 months. The contents of chlorogenic acid, geniposide and baicalin remained $>90 \%$.

Saline, SGF or SIF are commonly used as the mediums in drug dissolution studies. However, besides pepsin and trypsin, there are many kinds of enzymes and intrinsic factors in stomach and small intestine, which may also affect the metabolism of drugs. In this study, we prepared gastric lavage fluid and intestinal lavage fluid as the mediums of drug dissolution.

In gastric lavage fluid, for hard capsule, three key ingredients dissolved quickly. But baicalin degraded after 15 minutes, which might be caused by pepsin and $\mathrm{pH}$ of the environment [6]. For soft capsule, the $\mathrm{Q}$ of three ingredients kept growing in the whole sampling time.

In intestinal lavage fluid, for hard capsule, the $Q$ of 3 key ingredients reached $90 \%$ in 2 hours. But then, they all decreased to about $80 \%$. For soft capsule, sustained release behaviors were observed in 12 hours and the $Q$ of three ingredients has been maintained. The oily accessories may protect the ingredients from degradation caused by enzymes, and $\mathrm{OH}^{-}$. Besides, the sustained release of the drug may also increase the bioavailability, in previous study [2].

Compared with chemical drug, traditional Chinese medicine (or herbal medicine) is characteristic of multi-component with multitarget, which brings a great challenge for quality control [7]. Usually, some components are chosen as indicators for quality control, however, they can hardly express the information of the preparation fully. Pattern recognition analysis in combination with fingerprint can provide a visual, simple and valid method for quality control of traditional Chinese medicine [8]. Integrated evaluation based on pattern recognition analysis can achieve a comprehensive analysis for multidimensional information of traditional Chinese medicine and has been used on study of Chinese material medica [9,10], like producing area[11], taxa of medicinal plant [12] and quality control of traditional Chinese medicine injections [13].

PCA (Principal Component Analysis) is a method of multivariate statistical analysis for integrated evaluation. A collection of observations of possibly correlated variables are transformed in to a set of values of linear uncorrelated variables (usually, the number of these new variables is less than that of the original variables), which were considered as principle components. The first principal component could explain as much of the variability in the data as possible. Each subsequent component also has the highest variance with the constraint that it is orthogonal to the preceding components. Finally, an orthogonal basis set with uncorrelated vectors was obtained.

Consider a data matrix, $\mathrm{X}$, its rows is fulfilled with peak areas of chromatography at different time, 
its columns is described by different samples. For example, Eq 1 is our original observation matrix:

$\mathrm{X}=\left(\begin{array}{ccc}x_{11} & \cdots & x_{1 m} \\ \vdots & " & \vdots \\ x_{n 1} & \cdots & x_{m m}\end{array}\right)$

In this matrix, $\mathrm{m}$ random variables $x_{1}, x_{2} \ldots, x_{\mathrm{m}}$ form a vector of $\mathrm{m}$ dimensions. That is, $\mathrm{x}=\left(x_{1}, x_{2}, \ldots, x_{m}\right)^{T}$. Its mean vector is denoted by $\mu$, its covariance is denoted by $\Sigma$. Consider the following linear transformation:

$$
\left\{\begin{array}{l}
F_{1}=a_{11} x_{1}+a_{12} x_{2}+\cdots+a_{1 m} x_{m} \\
F_{2}=a_{21} x_{1}+a_{22} x_{2}+\cdots+a_{2 m} x_{m} \\
F_{m 1}=a_{m 1} x_{1}+a_{m 2} x_{2}+\cdots+a_{m m} x_{m}
\end{array}\right.
$$

It can be written as the product of matrix:

$\mathrm{F}=\mathrm{Ax}$

where $\mathrm{F}=\left(F_{1}, F_{2}, \ldots, F_{m}\right)^{\mathbb{T}}$, and

$A=\left(\begin{array}{ccc}a_{11} & \cdots & a_{1 m} \\ \vdots & \ddots & \vdots \\ a_{m 1} & \cdots & a_{m m}\end{array}\right)=\left(\begin{array}{c}a^{T} \\ \vdots \\ a_{m n}^{5}\end{array}\right)$

As mentioned above, PCA is considered as a linear transformation method which can transform original data to a new coordinate system. The greatest variance by some projection of the data comes to lie on the first coordinate (called the first principal component), the second greatest variance on the second coordinate, and so on [14]. Thus we know that the problem to obtain the principal component is to find a linear function of $x$ of variable $\mathrm{x}$ such that the variance of $a_{1}^{T} x$ could be as large as possible. That is, we need

$$
\begin{aligned}
\operatorname{var}\left(F_{i}\right)= & \operatorname{var}(\alpha T x)=\mathbb{E}\left\{\left[a\left[x-\mathbb{E}(\alpha[x)]^{2}\right]\right.\right. \\
& =a_{1}^{T} E\left\{[x-E(x)][x-E(x)]^{T}\right\} a_{i} \\
& =\alpha\left[\Sigma a_{i} \ldots \ldots \ldots \ldots(7)\right.
\end{aligned}
$$

reach its maximal value. And we have two constraint conditions. First, we need

$a_{1}^{T} a_{i}=1$

to avoid $\operatorname{var}\left(F_{i}\right) \rightarrow \infty$.

Second, we want each of principal components $F_{i}(i=1,2, \cdots, m)$ are uncorrelated. We need

$\operatorname{cov}\left(F_{i}, F_{j}\right)=\alpha I \Sigma a_{j}=0(i, j=1,2, \cdots, m, i \neq j)$
Under these constraint conditions, we know that principal components achieve their maximal value when $a_{i}$ is the eigenvector of $\Sigma$ and the eigenvalue of $\Sigma$ is the variance of principal components.

Assume there are $\mathrm{m}$ eigenvalues of $\Sigma$ : $\lambda_{1} \geq \lambda_{2} \geq \cdots \geq \lambda_{m} \geq 0$, and $\varepsilon_{1}, \theta_{2}, \cdots, \theta_{m}$ denotes corresponding normalized orthogonal eigenvectors. Its matrix have the form:

$\mathbf{E}=\left(\theta_{1}, \theta_{2}, \cdots, \theta_{\mathrm{m}}\right)=\left(\begin{array}{ccc}\epsilon_{11} & \cdots & \epsilon_{1 \mathrm{~m}} \\ \vdots & { }_{1} & \vdots \\ \epsilon_{m 1} & \cdots & \epsilon_{m m}\end{array}\right)$

Then the ith principal component is given by the following:

$F_{1}=e_{i}^{T} x=\epsilon_{1:} x_{1}+\epsilon_{2 i} x_{2}+\cdots \epsilon_{m i} x_{m}$

After getting the principal components, we can do some cluster analysis of our samples. Observe the crucial samples of each principal component, If $x_{1}$ and $x_{2}$ have superior contribution rate for $F_{1}$, and their contribution rate are close, we could conclude that they should be clustered. The computing process can be conducted by software SIMCA-P.

In our study of long-term stability, samples of soft capsules of different month were clustered together. Meanwhile, the samples of hard capsules were divided into two cluster, the samples of $6^{\text {th }}$ month are far from the other samples, which indicates that the fingerprint of hard capsule has changed a lot in $6^{\text {th }}$ month. To some extent, soft capsule is more stable than hard capsule.

In the study of drug dissolution evaluation, samples of different time were taken as the columns of the matrix. To understand which components contributed to the differentiation, a Loading Bi-plot, which presented all key items (samples of different time and chemical components represented by different peaks) in one picture to demonstrate the relationships among them, was investigated. In this plot, samples (represented by diamonds) in close proximity to components (represented by triangles) have high levels of these component, and the components away from the sample symbols are low in these samples.

In general, the proximity between the sample symbols and component symbols indicates their correlation. Therefore, this Bi-plot showed that most of the components (peaks) are positively correlated with the samples from $6 \mathrm{~h}$ to $24 \mathrm{~h}$, 
which indicated the sustained release behavior of the soft capsule. For soft capsule, an O/W emulsion may be formed by the protein in intestinal lavage fluid with gently shaking. The indicators (chlorogenic acid, geniposide and baicalin) dispersed in the oily accessories were kept stable and the other components were released slowly. Previous study showed that the bioavailability of baicalin in soft capsule is higher than that of hard capsule, which may be also caused by the protection and absorption enhancement of emulsion.

\section{CONCLUSION}

QKL soft capsule displayed better stability than the hard capsule both in vitro and in semi-bionic condition. The soft capsule also displayed sustained release behavior, and thus, may also have a superior therapeutic effect.

\section{DECLARATIONS}

\section{Acknowledgement}

This work was supported in part by Bejing Newstar Plan of Science and Technology (grant no. xx2015A048), Beijing Key Laboratory (grant no. BZ0102) and Study of Qingkailing soft and hard capsule Technology Development (grant no. 2014110031014019). Naiqi Song, a statistician at Beijing University of Chinese Medicine, assisted with the PCA work.

\section{Conflict of Interest}

No conflict of interest associated with this work.

\section{Contribution of Authors}

The authors declare that this work was done by the authors named in this article and all liabilities pertaining to claims relating to the content of this article will be borne by them.

\section{Open Access}

This is an Open Access article that uses a funding model which does not charge readers or their institutions for access and distributed under the terms of the Creative Commons Attribution License (http://creativecommons.org/licenses/by/ 4.0) and the Budapest Open Access Initiative (http://www.budapestopenaccessinitiative.org/rea d), which permit unrestricted use, distribution, and reproduction in any medium, provided the original work is properly credited.

\section{REFERENCES}

1. Ai CL, Xie YM, Li MQ, Wang $L X$, Liao X. Incidence rate of adverse reaction/event by Qingkailing injection: a Metaanalysis of single rate. Zhongguo Zhongyao Zazhi. 2015; 40(24): 4770-4778.

2. Zhang JY, Jiang YX, LiU H, Chen QY, Gai CH, Xu $H$. Pharmacokinetics of Baicalin from Qingkailing Hard Capsules and Soft Capsules in Rats. Journal of Yantai University, 2015; 28(2):103-107.

3. Ministry of Science and Technology of the People's Republic of China. The Guiding Principles for the Care and Use of Laboratory Animal. 2006. [Chinese]

4. China Drug Administration. The Technical Requirements of Fingerprint Research on Traditional Chinese Medicine Injection (TCMI) (interim). Chinese Traditional Patent Medicine. 2000; 22: 671-675. [Chinese]

5. Chinese Pharmacopoeia Commission. Chinese Pharmacopoeia. Beijing: China Medical Science Press; 2015. [Chinese]

6. Jie $X$, Chen $X$, Zhong $D$. Stability of baicalin in biological fluids in vitro. J Pharmaceut Biomed. 2005; 39(4): 593600.

7. Huang YP, Wu ZH, Su RH, Ruan GH, Du FY, Li GK Current application of chemometrics in traditional Chinese herbal medicine research. J Chromatogr $B$. 2016; 1026: 27-35.

8. Li N, Che $Y$, Zhang L, Zhang J, Zhou $Y$, Jiang $Y$. Fingerprint analysis of Ophiopogonis Radix by HPLCUV-ELSD coupled with chemometrics methods. J Chinese Pharmaceut Sci 2013; 22(1): 55-63.

9. Alaerts G, Merino-Arévalo $M$, Dumarey $M$, Dejaegher $B$, Noppe N, Matthijs N, Smeyers-Verbeke J, Vander Heyden $Y$. Exploratory analysis of chromatographic fingerprints to distinguish rhizoma chuanxiong and rhizoma ligustici. J Chromatogr A. 2010; 1217(49): 7706-7716.

10. Qu B, Hu YZ. Non-Negative Matrix Factorization-Based SIMCA Method to Classify Traditional Chinese Medicine by HPLC Fingerprints. J Chromatogr Sci. 2011; 1217(49): 189-197.

11. Fan $Q M$, Chen $C Y$, Huang $Z Q$, Zhang $C M$, Liang $P J$, Zhao SL. Discrimination of Rhizoma Gastrodiae (Tianma) using 3D synchronous fluorescence spectroscopy coupled with principal component analysis. Spectrochim Acta A. 2014; 136: 1621-1625.

12. Ma J, Qi LW, Li HJ, Li P. A segmental monitoring strategy based on variable wavelength detection for quality control of three Polygonaceae herbs. J Pharmaceut Biomed. 2012; 62(2): 155-161.

13. Sun $L Q$, Wang $S Y$, Li YJ, Wang $Y X$, Wang ZZ, Huang WZ, Wang YS, Bi YA, Ding G, Xiao W. Impact of parameter fluctuations on the performance of ethanol precipitation in production of Re Du Ning Injections, based on HPLC fingerprints and principal component analysis. Chin J Nat Med. 2016; 14(1): 73-80. 
Sun et al

14. Chen $X D$, Chen $C$, Jin L. Principal component analyses

14. in anthropological genetics. Adv Anthropol 2011; 1(2): 9- 